\title{
Unprecedented loss of ammonia assimilation capability in a urease-encoding bacterial mutualist
}

\author{
Laura E Williams ${ }^{1}$, Jennifer J Wernegreen ${ }^{1,2^{*}}$
}

\begin{abstract}
Background: Blochmannia are obligately intracellular bacterial mutualists of ants of the tribe Camponotini. Blochmannia perform key nutritional functions for the host, including synthesis of several essential amino acids. We used Illumina technology to sequence the genome of Blochmannia associated with Camponotus vafer.

Results: Although Blochmannia vafer retains many nutritional functions, it is missing glutamine synthetase $(\mathrm{g} / \mathrm{n} A)$, a component of the nitrogen recycling pathway encoded by the previously sequenced $B$. floridanus and $B$. pennsylvanicus. With the exception of Ureaplasma, B. vafer is the only sequenced bacterium to date that encodes urease but lacks the ability to assimilate ammonia into glutamine or glutamate. Loss of glnA occurred in a deletion hotspot near the putative replication origin. Overall, compared to the likely gene set of their common ancestor, 31 genes are missing or eroded in B. vafer, compared to 28 in B. floridanus and four in B. pennsylvanicus. Three genes (queA, vis $C$ and yggS) show convergent loss or erosion, suggesting relaxed selection for their functions. Eight $B$. vafer genes contain frameshifts in homopolymeric tracts that may be corrected by transcriptional slippage. Two of these encode DNA replication proteins: dnaX, which we infer is also frameshifted in B. floridanus, and dnaG.

Conclusions: Comparing the B. vafer genome with B. pennsylvanicus and B. floridanus refines the core genes shared within the mutualist group, thereby clarifying functions required across ant host species. This third genome also allows us to track gene loss and erosion in a phylogenetic context to more fully understand processes of genome reduction.
\end{abstract}

\section{Background}

Candidatus Blochmannia species (hereafter, Blochmannia) are obligately intracellular, primary mutualists of ants belonging to the tribe Camponotini [1,2]. Blochmannia have been found in every species of Camponotus surveyed to date, as well as other genera within Camponotini, suggesting an ancient and stable association with the ant tribe [3]. The nutritional basis of the symbiosis was first suggested by the location of Blochmannia in specialized host cells intercalated among epithelial cells in the insect midgut $[4,5]$. Genome sequencing of $B$. floridanus and $B$. pennsylvanicus revealed biosynthetic pathways for all essential amino acids except arginine, further supporting the hypothesis

\footnotetext{
* Correspondence: j.wernegreen@duke.edu

${ }^{1}$ The Institute for Genome Sciences and Policy, Duke University, Durham, NC, USA

Full list of author information is available at the end of the article
}

that Blochmannia provides important nutritional functions to the ant host [6,7]. The symbiont also recycles nitrogen from urea into glutamine via the activity of urease and glutamine synthetase [8], which are encoded by the two previously published Blochmannia genomes $[6,7]$. Urease hydrolyzes urea to ammonia and carbon dioxide, and glutamine synthetase assimilates nitrogen from ammonia into glutamine, which feeds into other amino acid biosynthetic pathways [9]. This process may consume the nitrogenous waste products of the ant host and also urea in animal or bird waste collected and ingested by some Camponotus species [8].

Similar to other primary obligate mutualists of insects, Blochmannia genomes are extremely reduced in size (705-792 kb), show strong AT bias (27-29.5\% GC content), and undergo accelerated rates of molecular evolution $[6,7,10]$. Comparative analyses of Buchnera, the primary mutualist of aphids, suggest that ongoing
C Biomed Central

(c) 2010 Williams and Wernegreen; licensee BioMed Central Ltd. This is an Open Access article distributed under the terms of the Creative Commons Attribution License (http://creativecommons.org/licenses/by/2.0), which permits unrestricted use, distribution, and reproduction in any medium, provided the original work is properly cited. 
genome reduction proceeds primarily as a gradual erosion of individual genes, rather than by punctuated losses of large DNA segments encoding multiple genes [11]. Comparisons of the B. floridanus and B. pennsylvanicus genomes indicate a similar phenomenon in Blochmannia [7]. The accumulation of substitutions, small indels and frameshift errors slowly degrades genes, and the resulting pseudogenes are gradually eliminated from the genome by the underlying deletional bias in bacteria [12]. The complement of genes lost by an endosymbiont genome is determined in part by chance events, such as the fixation of deleterious mutations due to small effective population sizes, and by the historical contingencies of gene loss in ancestral genomes, which influence the strength of selective pressure on remaining genes [13]. Independent loss of the same gene from multiple lineages would suggest relaxed selection on gene function, rather than stochastic effects of drift.

The AT mutational bias observed in obligate intracellular symbionts results in a high density of homopolymeric tracts of adenines or thymines in the genome, with some tracts as long as $12 \mathrm{bp}$. These polyA or poly $\mathrm{T}$ tracts are prone to polymerase slippage during replication, which can introduce frameshift errors in the gene. In some cases, such frameshifts may constitute the initial events of gene erosion, leading to accumulation of mutations and eventual degradation to a pseudogene [14]. However, some frameshifts are conserved among geographically disparate symbiont populations and even between different species, with no detectable degradation of the gene $[15,16]$. Full-length, functional protein products are generated from these frameshifted genes as a result of transcriptional slippage in the homopolymeric tract, which produces a mixed mRNA pool of full-length and frameshifted transcripts [15].

To better understand the nutritional symbiosis between Blochmannia and Camponotini and processes of genome reduction in this endosymbiont, it is essential to compare multiple genomes. Expanding the set of completed Blochmannia genomes may shed light on the 'core' genes shared across Blochmannia and refine our knowledge of the functions that are required in this symbiosis. Comparing multiple genomes within this group can also clarify the Blochmannia pan-genome and let us reconstruct the likely gene content of ancestral genomes, thereby elucidating patterns of gene loss and erosion. Finally, analysis of Blochmannia genomes from different Camponotus host species may help us understand how this symbiosis evolves in diverse hosts that occupy different habitats and geographic ranges.

Here, we use Illumina technology to sequence the genome of Blochmannia vafer, the endosymbiont of Camponotus vafer. The addition of this genome to the two previously sequenced Blochmannia genomes lets us investigate genomic changes in a phylogenetic context. Also, C. vafer occupies a different habitat and geographic range compared to $C$. floridanus and $C$. pennsylvanicus. Whereas $C$. pennsylvanicus is widespread throughout much of the eastern and central United States, and C. floridanus is distributed throughout the southeastern United States as well as Central and South America [17], C. vafer is found in a more restricted range in the southwestern United States and northeastern Mexico [18]. Completion of the B. vafer genome allows us to investigate the nature of the mutualism in ecologically distinct Camponotus species.

\section{Results}

Shared Blochmannia genes include urease gene cluster

The $B$. vafer genome shares many features with the $B$. pennsylvanicus and B. floridanus genomes, including reduced size and low \%GC content (Table 1). Genes shared among the three Blochmannia include 617 intact genes, 575 of which are protein-coding (Figure 1). For the purposes of these comparisons of functional capabilities, we include as 'intact' those genes that contain a single indel within a homopolymeric tract, since the reading frame may be restored via transcriptional slippage [15]. B. vafer, B. floridanus and B. pennsylvanicus encode the same set of DNA replication proteins and ribosomal proteins, with the exception of the $50 \mathrm{~S}$ ribosomal protein RpmD, which is absent in B. pennsylvanicus. All three Blochmannia encode the same pathways for essential amino acid biosynthesis and sulfate assimilation, the latter of which contributes to cysteine biosynthesis. A urease gene cluster, which includes the structural genes ure $A B C$ and the accessory genes

Table 1 Blochmannia genome statistics

\begin{tabular}{lccc}
\hline & B. vafer & B. floridanus & B. pennsylvanicus \\
\hline Size (bp) & 722,593 & 705,557 & 791,654 \\
GC content & $27.5 \%$ & $27.4 \%$ & $29.6 \%$ \\
Total genes & 631 & 637 & 659 \\
Protein-coding genes & 587 & $590^{1}$ & 610 \\
tRNA & 37 & 37 & $40^{2}$ \\
rRNA & 3 & 3 & 3 \\
Other RNA & 2 & $3^{3}$ & 33 \\
Pseudogenes & 2 & 4 & 3 \\
Average CDS length & 1,006 & 1,002 & 995 \\
Median IGS length & 134 & 114 & 172 \\
Percent protein-coding & $81.7 \%$ & $83.9 \%$ & $76.7 \%$ \\
Percent coding (inc. RNA) & $82.9 \%$ & $85.0 \%$ & $77.8 \%$
\end{tabular}

${ }^{1}$ Gene counts for B. floridanus include four protein-coding genes not identified in the original annotation that were detected by comparisons with $B$. vafer and $B$. pennsylvanicus.

${ }^{2}$ Re-analysis of the $B$. pennsylvanicus genome revealed that the Gly-GGA tRNA pseudogene reported earlier is likely functional.

${ }^{3}$ We identified a RNA-coding gene in B. floridanus and B. pennsylvanicus that was not previously annotated. 


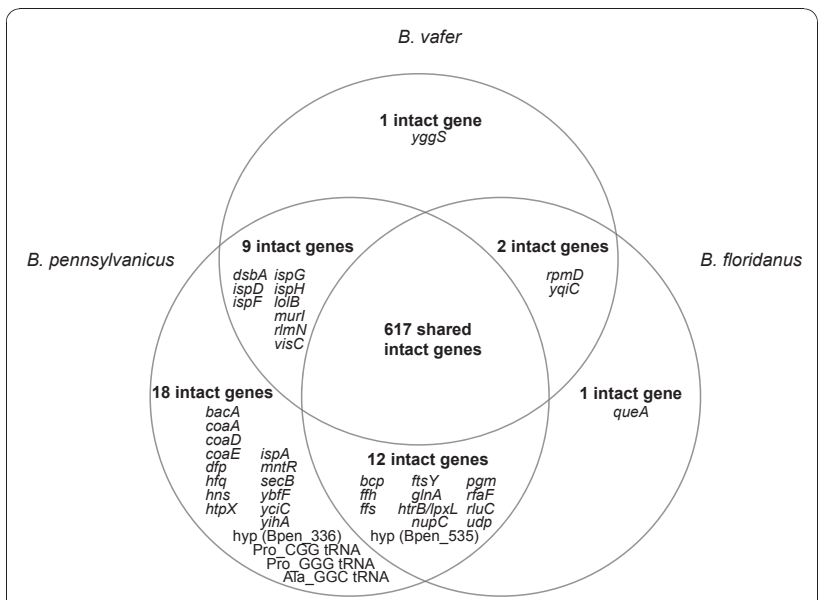

Figure 1 Comparison of intact genes encoded by three Blochmannia genomes. To highlight potential metabolic differences among genomes, only intact genes are shown. These include protein-coding and RNA-coding genes. Eroded pseudogenes in a given genome are considered missing due to loss of function. Genes with a single frameshift in a polyA or polyT tract are considered intact for this purpose, because transcriptional slippage may restore expression of a full-length protein product. In this comparison, yidC and yidD in B. floridanus are counted as a single gene, due to fusion in B. pennsylvanicus and $B$. vafer.

ureDFG [19], is also present in all three genomes. Gene order and strand orientation is strictly conserved along all three Blochmannia genomes. The set of genes encoded by each Blochmannia genome is listed in Additional File 1.

\section{Loss of glutamine synthetase in $B$. vafer}

The most recent common ancestor of all three Blochmannia had at least 660 genes (Figure 1). This reconstruction considers only genes that are intact in at least one of the genomes (the $u v r D$ pseudogene found in B. pennsylvanicus is therefore not included). At least 31 genes were lost or eroded along the branches leading from the common ancestor to B. vafer, compared to 28 genes for B. floridanus and four for B. pennsylvanicus. Twelve genes are missing or eroded only in $B$. vafer (Figure 1). Of these, glutamine synthetase $(g \ln A)$ is perhaps the most startling. We confirmed the absence of $g \ln A$ in Blochmannia from eight additional C. vafer colonies by Sanger sequencing of this region. The sequences were identical, with the exception of one substitution and three indels. Analysis of the $\operatorname{trp} E$ gene encoded by $B$. vafer revealed three distinct genotypes, demonstrating that $g \ln A$ is missing from different B. vafer strains.

In other Blochmannia species, Class I glutamine synthetase is thought to act in concert with the urease gene cluster (ureDABCFG) to recycle nitrogen into amino acid biosynthesis [8]. Urease converts urea to carbon dioxide and ammonia, the latter of which is then assimilated into glutamine by glutamine synthetase. Despite losing $g \ln A, B$. vafer retains ureDABCFG, which have high amino acid sequence identity (53-80\%) to the $B$. floridanus urease proteins. Loss of glutamine synthetase in B. vafer disrupts the ammonia assimilation step of nitrogen recycling. In $E$. coli, glutamate dehydrogenase $(g d h A)$ encodes an alternative ammonia assimilation pathway [20], but none of the sequenced Blochmannia retain this gene. We searched the NCBI Genomes database to determine whether any other completely sequenced bacterium possesses urease but lacks glutamine synthetase and glutamate dehydrogenase. Of the 371 bacterial genomes encoding urease, 359 also have an ortholog of the $B$. pennsylvanicus Class I glutamine synthetase. Among the remaining 12 genomes, four have an ortholog of $g \ln A$ from Bacteroides fragilis, which is the prototypical example of a Class III glutamine synthetase, and five genomes have an ortholog of E. coli glutamate dehydrogenase $(g d h A)$. In all, 368 of the 371 bacterial genomes with urease also encode an ammonia assimilation pathway catalyzed by either glutamine synthetase (Class I or Class III) or glutamate dehydrogenase. The three genomes lacking $g \ln A$ or $g d h A$ are all strains of Ureaplasma, which uses high intracellular concentrations of ammonia to generate an electrochemical gradient for driving ATP synthesis [21].

\section{The urease gene cluster in Blochmannia}

To determine whether loss of glutamine synthetase is correlated with relaxed selection on the urease genes in $B$. vafer, we compared evolutionary rates along the lineages leading to $B$. vafer and $B$. floridanus since their divergence from a common ancestor. For the 570 orthologs analyzed, the fold-increase in evolutionary rate along the lineage leading to $B$. vafer had a wide range $(0.1$ - 4.6), but a median value of 1.04 , indicating no systematic rate difference between the $B$. vafer and B. floridanus lineages (Additional File 2). At urease genes, the rate increase in $B$. vafer varied from 0.53 - 1.87 for the three structural genes (ureABC) and 0.96 - 1.19 for the accessory genes (ureDFG), indicating that the absence of $g \ln A$ in $B$. vafer is not correlated with consistent rate acceleration in the urease proteins.

We found no evidence to suggest that the urease operon in Blochmannia was acquired by horizontal gene transfer from outside of the gamma-proteobacteria. Blochmannia urease proteins were most similar by BLASTP analysis to those of other gamma-proteobacteria, including Pseudomonas (e.g., NC_010501) and Klebsiella (e.g., NC_009648). Gene order in the urease gene cluster was conserved among the related free-living bacteria and Blochmannia; however, the Pseudomonas urease operon consists of eight genes (ureDABCEJFG), 
whereas the Blochmannia urease operon lacks urease accessory genes ureE and ureJ. For all three sequenced Blochmannia, the intergenic sequence (IGS) between ure $C$ and ureF is $298-470 \mathrm{bp}$, much larger than the other IGSs in this operon, which ranged from $2-122$ bp. As described below, the longer IGSs suggest that $u r e E$ and $u r e J$ might have been present in the ancestor of Blochmannia.

\section{Processes of genome reduction in $B$. vafer (i) Deletion hotspot near the origin of replication}

Genes missing or eroded in B. vafer were generally scattered throughout the genome, with the exception of eight missing genes near the putative origin of replication (Figure 2). Because of the absence of a characteristic cluster of $d n a A$ boxes, we identified the origin of replication based on the shift in GC skew occurring between $y i b N$ and hldD (Figure 3). Similar to B. florida$n u s$, the putative origin of $B$. vafer is located approximately $32 \mathrm{~kb}$ upstream of gidA, which is the origin of replication of B. pennsylvanicus [6,7]. This $32 \mathrm{~kb}$ region contains three genes that are missing from both $B$. vafer and $B$. floridanus and were likely lost prior to their divergence, another gene missing from $B$. floridanus only, and four genes missing from $B$. vafer only, including $g \ln A$.

\section{(ii) Frameshifts within homopolymeric tracts}

Similar to other obligate insect endosymbionts, the genome of B. vafer is extremely AT-biased (Table 1). All three Blochmannia genomes contain numerous long (9 or more bp) polyA tracts within protein-coding regions (153 such tracts in B. vafer, compared to 134 in B. floridanus and 114 in B. pennsylvanicus). Indels in homopolymeric tracts and resulting frameshifts are considered key events in gene inactivation and loss in endosymbiont genomes [14]. However, some frameshifts may be corrected by polymerase slippage during transcription, allowing expression of full-length proteins $[7,15,16]$. Eight $B$. vafer genes have short indels within polyA or polyT tracts resulting in frameshifts, compared to four such genes in B. floridanus and four in B. pennsylvanicus (Figure 4). For the four genes that were frameshifted exclusively in $B$. vafer (dnaG, dxs, pth and ribF), we found no evidence of unusually accelerated evolutionary rates along the lineage leading to $B$. vafer. Specifically, when compared to the lineage leading to B. floridanus, the fold-increase in evolutionary rates at the four genes in B. vafer ranged from 1-1.5, well within the distribution for the 570 orthologs tested. Although it is conceivable that these frameshifts are recent, it is also possible that the reading frame is corrected via transcriptional slippage, as demonstrated for other Blochmannia and Buchnera genes $[7,15,16]$.
Of the eight $B$. vafer genes with frameshifts, three are involved in information transfer: dnaX, dnaG and $p t h$. DnaX encodes two subunits of the DNA polymerase III holoenzyme, which is the primary replicative enzyme in bacteria. In $E$. coli, expression of full-length $d n a X$ generates the tau subunit, whereas ribosomal slippage generates the shorter gamma subunit [22-24]. Both of these subunits are thought to participate in assembly of the beta sliding clamp, which affects processivity of the pol III holoenzyme [25]. The dnaX frameshift reported here occurs downstream of the ribosomal slippage site, within the region exclusive to the tau subunit. Comparisons of $B$. vafer and B. floridanus allowed us to identify the same frameshift in $B$. floridanus dnaX, which was previously reported as truncated [6]. Because this frameshift may be corrected by transcriptional slippage, we propose that expression of full-length $d_{n a X}$ tau subunit occurs at some level in both B. vafer and B. floridanus. This frameshift is not found in B. pennsylvanicus dnaX, possibly because the polyA tract is interrupted by two thymines, which reduces the chances of slippage during replication or transcription (Figure 4). The two other frameshifted information transfer genes in $B$. vafer encode DNA primase (dnaG) and peptidyl-tRNA hydrolase (pth) (Figure 4). DnaG synthesizes short RNA primers essential for lagging strand DNA replication, whereas Pth plays an important role in maintaining efficient protein synthesis by cleaving peptidyl-tRNAs released from stalled ribosomes [26].

To provide a phylogenetic perspective on these indels within homopolymeric tracts, we confirmed that $B$. vafer and $B$. floridanus are more closely related to each other than either is to B. pennsylvanicus (Additional File 3). Based on this topology, two genes, erfK and $y t f M$, show evidence for independent frameshift events and/or a reversion to an intact reading frame (Figure 5). Independent frameshift events in $y t f M$ are supported by the different location of the indel in B. pennsylvanicus, compared to B. floridanus and B. vafer (Figure 4).

\section{(iii) Convergent patterns of gene loss or erosion among Blochmannia}

In addition to examining the history of frameshifted genes, we also identified several examples of convergent gene loss and erosion among the three Blochmannia genomes (Figure 5). Three genes (queA, visC and yggS) were likely lost or eroded in independent events. QueA was likely lost in the lineage leading to $B$. pennsylvanicus and degraded in the lineage leading to $B$. vafer sometime after $B$. vafer and B. floridanus diverged. Vis $C$ is intact in $B$. pennsylvanicus but absent from B. floridanus. In $B$. vafer, vis $C$ has a single indel within a tract of 11 adenines. This frameshift might be corrected by transcriptional slippage, but it could, in principle, also 

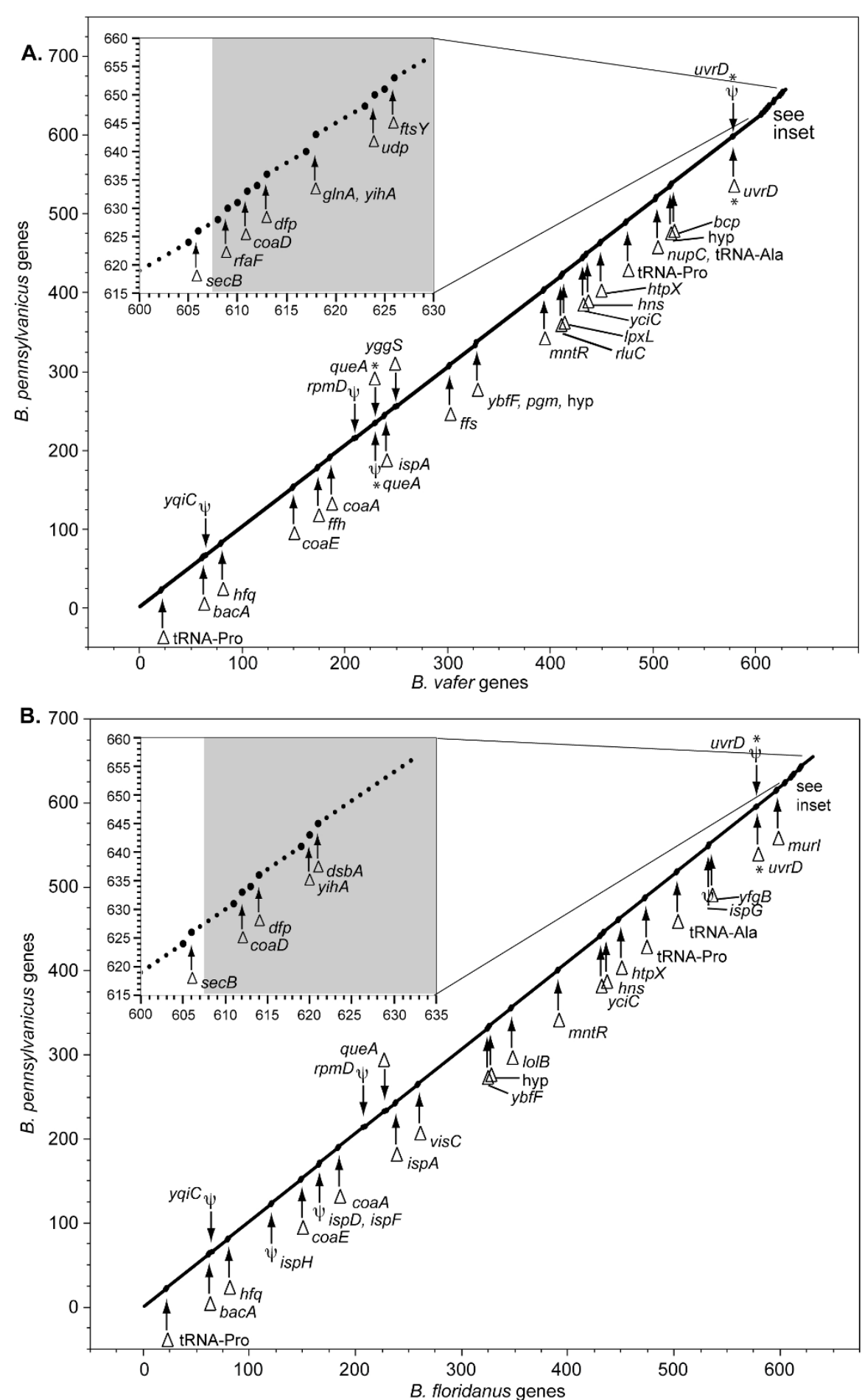

Figure 2 Gene loss in B. vafer and B. floridanus compared to B. pennsylvanicus. (a) B. vafer genes were plotted against orthologs in $B$. pennsylvanicus. The $x$ - and $y$-axes correspond to gene number in $B$. vafer and B. pennsylvanicus, respectively. Locations of missing genes (represented by a delta symbol) and eroded genes (represented by a psi symbol) are emphasized by larger dots. Gene names are listed next to the symbols. Two genes, marked with asterisks, are missing in one of the genomes and present only as a pseudogene in the other genome. The inset shows a more detailed view of the $\sim 30 \mathrm{~kb}$ region in $B$. vafer near the putative replication origin, in which eight genes are missing compared to $B$. pennsylvanicus. The grey shaded region in the inset indicates the relocation of the origin in $B$. vafer compared to $B$. pennsylvanicus. (b) B. floridanus genes were plotted against orthologs in B. pennsylvanicus, using the same methodology as above. 


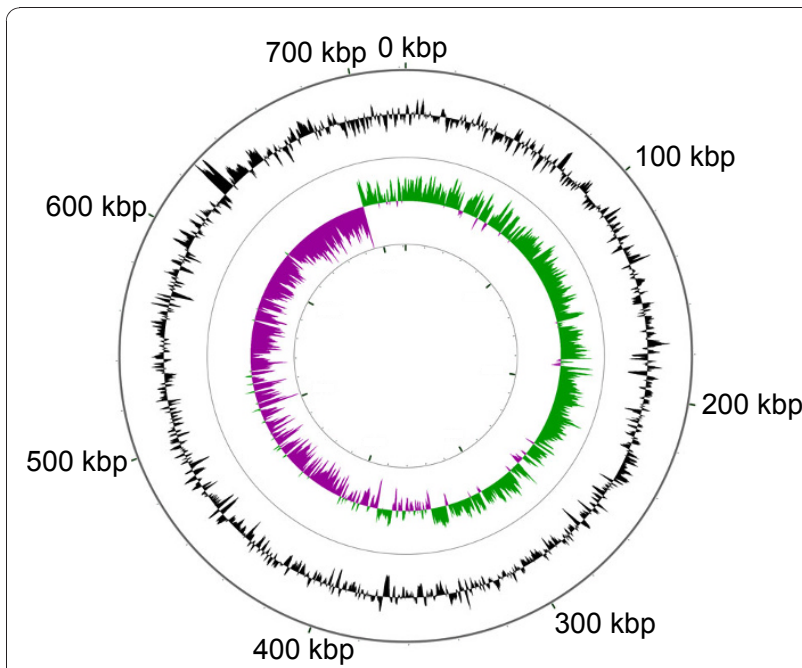

Figure $3 \mathrm{GC}$ content and GC skew of $B$. vafer genome. GC content (black) and GC skew (green and purple) of the B. vafer genome. For GC skew, the center line indicates the average GC skew value for the genome. Green shading above the line denotes GC skew values greater than the genome average, whereas purple shading below the line denotes GC skew values less than the genome average. Major and minor tick marks on the outermost and innermost circles show nucleotide position on the genome in 100 $\mathrm{kbp}$ and $20 \mathrm{kbp}$ increments, respectively.

represent very recent gene erosion that is independent from vis $C$ loss in $B$. floridanus.

In the case of $y g g S$, no detectable relicts of the gene remain in either $B$. floridanus or B. pennsylvanicus, but the gene is intact in $B$. vafer. We found no evidence to support the hypothesis that $y g g S$ was acquired by B. vafer via horizontal gene transfer. The top BLASTP hits for B. vafer YggS were other gamma-proteobacteria, including the insect endosymbiont Sodalis glossinidius, which is considered a close relative of Blochmannia [27]. In addition, the corresponding intergenic spacer sequences in B. floridanus (663 bp) and B. pennsylvanicus (942 bp) were much larger than median IGS lengths (114 bp and $172 \mathrm{bp}$, respectively; see below), suggesting the presence of a severely eroded gene remnant. Finally, the GC content and codon usage of $y g g S$ is typical for the B. vafer genome (data not shown). Combined, this data strongly argues against recent horizontal transfer and are most consistent with yggS being present in the ancestral Blochmannia genome. This gene was most likely lost independently in the lineage leading to $B$. floridanus, sometime after B. floridanus and B. vafer diverged, and in the lineage leading to $B$. pennsylvanicus.

YggS might be involved in osmotolerance [28]; however, its exact function is unclear [29]. Notably, this protein is encoded by a number of other bacterial endosymbionts of insects (Figure 6), including Blattabacterium (cockroach), Buchnera aphidicola (aphid),

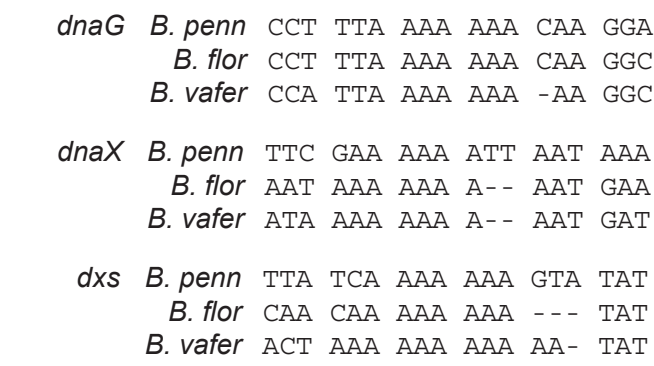

erfKlybiS B. penn ACA CAT AAA A-- AAA AAT

B. flor AAA AAT AAA AA- AAA AAT

B. vafer $\mathrm{AAA}$ GAT AAA AAA AAA GAT

gmhB B.penn AAT AAT TTT TTT TTC ATT B. flor GAT AAT TTT TTT TT- ATT

B. vafer AAT AAT TTT TTT TT- ATT

hisH B. penn CAA TTA AAA AAA AAA A-T CTA B. flor CAA TTG AAA AAA AAA AAT CTC

B. vafer CAA CTA GAA AAA ACC AAT CTT

pth B.penn TAT GAG AAA TAT AAG ATT B. flor CAT GAA GGT ATT CAG ATT

B. vafer CAT GAA AAA AAA AA- ATT

ribF B. penn CAA AAA AAA CGA AAC ATT B. flor AAT AAA AAG AAA ATC AAT

B. vafer TGT GAA AAA AAA A-- AAT

ubiF B. penn ATG AAA AAA AAA A- - - - - CCG B. flor ACC ATG AAT AAT AGT ATT TTA CCA B. vafer AAA AAG AAT AAA AAT ACT ATA CCA

visC B.penn ATT GTT -.. - - - - - TTC GAT B. flor gene is missing

B. vafer ATA TTA AAA AAA AAA A-- TTT GAA

ytfM B. penn GAT TT- AAA AAA AAA ATA B. flor GAT ATT AGT AAT CAA ATA B. vafer AAT TTA AAA AAT GAA ATA

ytfM B.penn TTT CAA CAA AAA AAA AAA ATA B. flor TGT - - - - - AA AAA AAA ACT

B. vafer TGT GCG -AA AAA AAA AAA TTA

Figure 4 Frameshifts in polyA or polyT tracts in Blochmannia genes. Alignments illustrate frameshifted regions of the eleven genes that contain indels within homopolymeric tracts in one or more Blochmannia genomes. Apart from the frameshifts shown, these genes are otherwise in frame. The frameshifts may be corrected by transcriptional slippage to yield full-length, in-frame transcripts.

Hamiltonella defensa (aphid), Sodalis glossinidius (tsetse fly), and Wigglesworthia glossinidia (tsetse fly). Of those endosymbionts lacking yggS, a few have severely reduced genomes, such as Carsonella (160 kb), Hodgkinia (144 kb) and Sulcia (244-277 kb).

Although the three Blochmannia genomes are severely reduced, most genome reduction occurred before their divergence. Ongoing gene disruption and loss since they diverged has affected $<10 \%$ of genes encoded in the ancestral genome. Given this, the probability that 


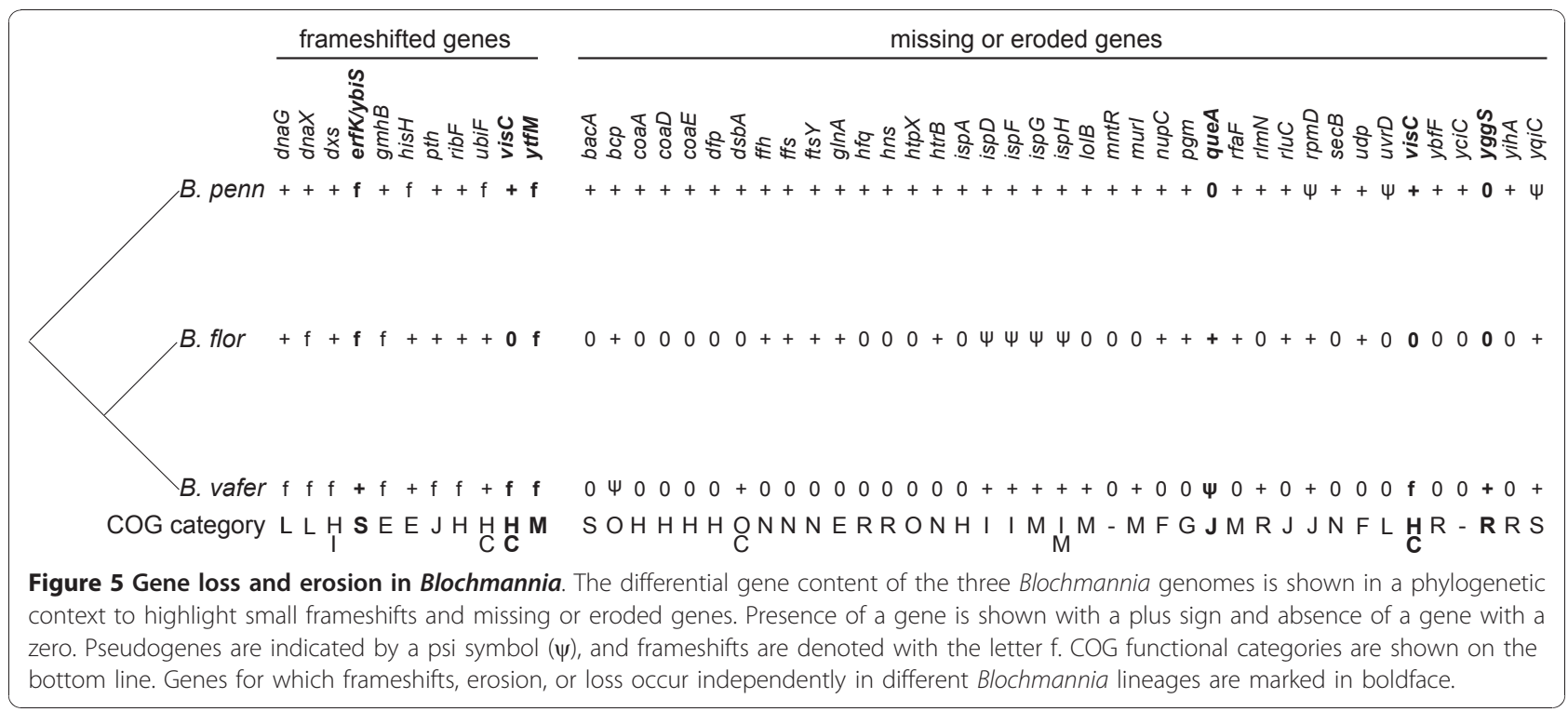

continued gene erosion and loss would occur by chance in two different Blochmannia lineages at the same gene is rather low. Instead, independent loss or erosion, such as we observed at queA and $y g g S$, suggests relaxed selective pressure on gene function. Conversely, the retention of queA and $y g g S$ in B. floridanus and B. vafer, respectively, suggests that their functions may remain important for host-specific aspects of the symbiosis.

\section{(iv) Longer intergenic sequences mark locations of gene losses}

Intergenic sequences in B. vafer and B. floridanus were significantly shorter than those of $B$. pennsylvanicus (Wilcoxon rank sum test; p < 0.001) (Table 1). Because gene order is strictly conserved in all three sequenced Blochmannia genomes, we were able to identify IGSs spanning missing genes in each genome. For both $B$. vafer and B. floridanus, such IGSs were significantly longer than IGSs between conserved genes (Wilcoxon rank sum test; $\mathrm{p}<0.0001$ ). We detected no significant difference in the lengths of IGSs spanning missing genes between $B$. vafer and $B$. floridanus (Wilcoxon rank sum test; $\mathrm{p}>0.05$ ), suggesting that deletion rates in these IGSs are similar in both genomes.

The importance of intergenic sequences in reduced endosymbiont genomes is largely unexplored. Coding sequences, including protein-coding and RNA-coding genes, occupy from $77.8-85.0 \%$ of the three Blochmannia genomes (Table 1), a lower fraction than many freeliving bacteria [12]. Non-coding elements within IGSs may play important roles in fundamental cellular processes. We searched the Blochmannia genomes for long palindromic sequences, which may impact control of transcription or mRNA stabilization [30]. We identified 20 large ( $30 \mathrm{bp})$ palindromes in B. floridanus, 10 in
B. vafer and 6 in B. pennsylvanicus; all but two were located at least partially in IGSs. Palindrome lengths ranged from 64 - $136 \mathrm{bp}$ in B. vafer (median $89 \mathrm{bp}$ ), including four exceptionally long palindromes $>100 \mathrm{bp}$ in length. B. floridanus and B. pennsylvanicus palindromes ranged from 62 - 104 bp (median 76 bp) and 62 $78 \mathrm{bp}$ (median $72 \mathrm{bp}$ ), respectively. Given the high rates of nucleotide and indel mutations in Blochmannia [31], intergenic palindromes would likely decay in the absence of selection favoring their presence.

\section{Discussion}

Like B. floridanus and B. pennsylvanicus, B. vafer retains several key nutritional functions that may benefit the ant host, including biosynthesis of all essential amino acids except arginine. However, the nitrogen recycling pathway proposed for B. floridanus and B. pennsylvanicus is not complete in B. vafer (Figure 7). Although $B$. vafer encodes the urease gene cluster, which hydrolyzes urea to ammonia and carbon dioxide, it lacks glutamine synthetase or any other means of assimilating nitrogen from ammonia into glutamine, an important precursor to biosynthesis of other amino acids.

In $B$. vafer, ammonia might be exported into the host cell cytosol, where Class II glutamine synthetase encoded by the ant host [32] may catalyze the assimilation of nitrogen and prevent the toxic accumulation of ammonia (Figure 7). Alternatively, excess ammonia may be used as a nitrogen donor by carbamoyl phosphate synthase (carAB). This enzyme, along with argI, catalyzes the initial steps of arginine biosynthesis in all three sequenced Blochmannia (Figure 7). The resulting product, citrulline, is exported to the host cell cytosol to complete arginine biosynthesis [6]. Although glutamine 


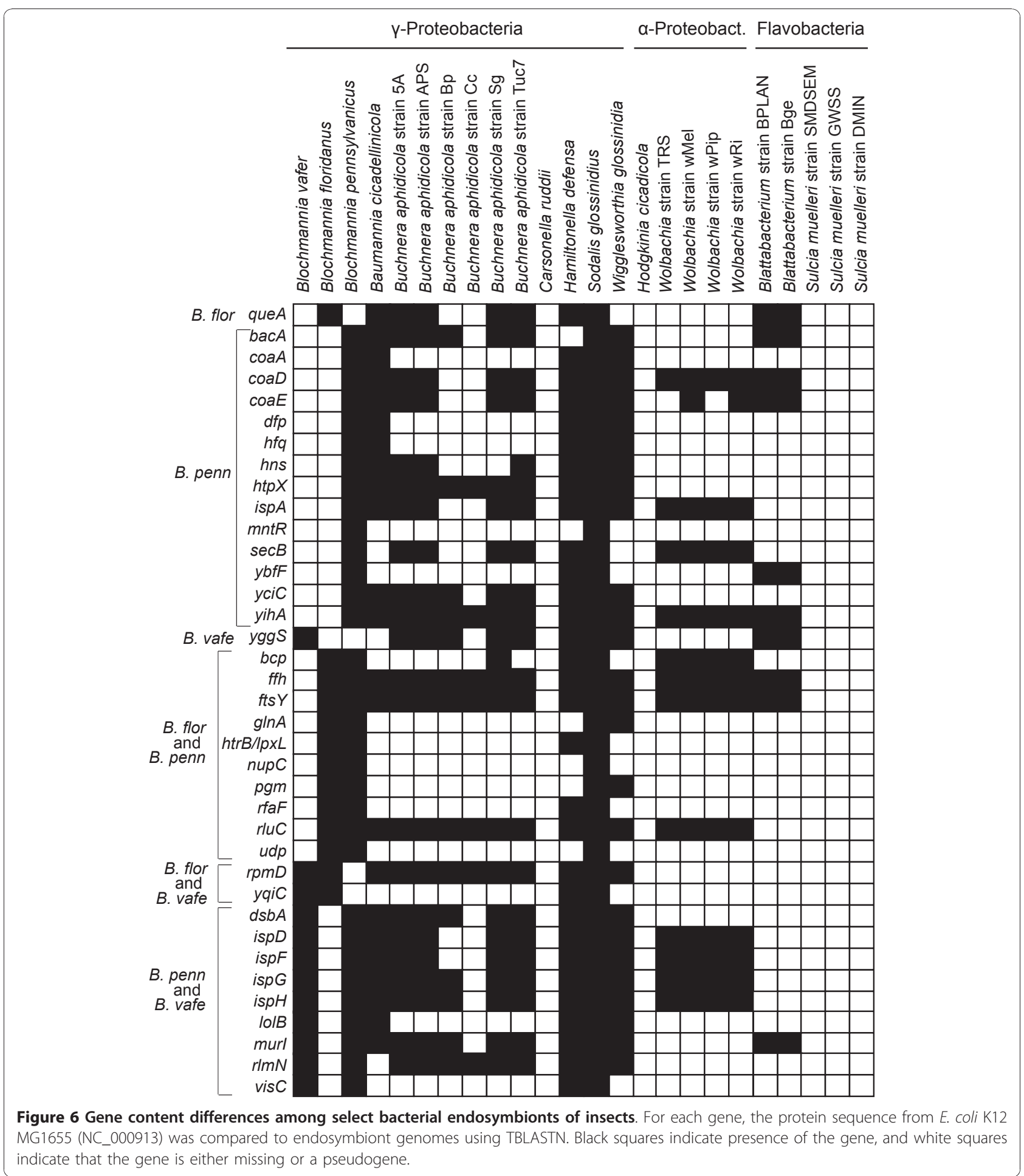

is the preferred nitrogen donor for $\operatorname{car} A B$, ammonia can be used as a substrate in the absence of glutamine [33]. Whether carbamoyl phosphate synthase plays a role in eliminating ammonia in B. vafer depends upon the concentration of glutamine in the endosymbiont cells (i.e., the amount of glutamine imported from the ant host). If glutamine is limiting, then $\operatorname{car} A B$ may use the excess ammonia produced by urease as a substrate.

With the exception of Ureaplasma, B. vafer is the only completely sequenced bacterium in the current NCBI Genomes database that possesses urease but lacks the ability to assimilate ammonia into glutamine or 


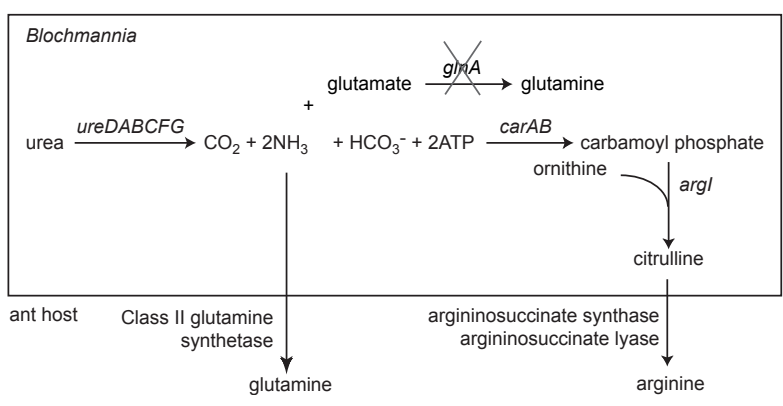

Figure 7 Possible alternative pathways for ammonia assimilation in B. vafer. Three possible pathways for ammonia assimilation in Blochmannia are shown. Glutamine synthetase $(g \ln A)$ is crossed out because of the absence of $g \ln A$ in $B$. vafer.

glutamate. All other bacteria with urease also encode ammonia assimilation pathways catalyzed by either glutamine synthetase (Class I or, more rarely, Class III) or glutamate dehydrogenase. This includes the cockroach endosymbiont Blattabacterium, which encodes urease and glutamate dehydrogenase $[34,35]$. The only exceptions are strains of the bacterial genus Ureaplasma, a commensal and opportunistic pathogen that colonizes the human urogenital tract and belongs to the Class Mollicutes [36]. In Ureaplasma, high intracellular concentrations of ammonia are thought to generate an electrochemical gradient that drives ATP synthesis [21]. There is no evidence that this process occurs in Blochmannia. With the loss of glutamine synthetase, $B$. vafer may have relinquished a component of the ancestral mutualism to the ant host. It is unclear whether or how the loss of $g \ln A$ affects the fitness of $B$. vafer and/or its ant host.

Gene loss and erosion is accelerated in the lineages leading to $B$. vafer and $B$. floridanus compared to $B$. pennsylvanicus. The mechanism(s) underlying the more extensive genome reduction in B. vafer and B. floridanus may occur at both the level of the endosymbiont and the level of the ant host. For example, the smaller size of intergenic spacers in B. vafer and B. floridanus suggest a faster rate of deletion mutations compared to the lineage leading to $B$. pennsylvanicus. Elevated mutation may reflect differences in the natural history of the host species sampled. Whereas C. pennsylvanicus and relatives in the subgenus Camponotus typically live in temperate regions and hibernate for part of the year, C. vafer and C. floridanus and their relatives live in warmer climates and their colonies are more active throughout the year. These differences in host activity and life history could affect the number of symbiont replications per year and thus influence the mutation rate per unit time.
Many genes missing in one or more Blochmannia genomes are also missing from other insect endosymbionts (Figure 6), suggesting that the functions encoded are not essential across all intracellular mutualists. Five genes showed evidence of independent instances of gene loss, erosion and/or frameshifts within Blochmannia. Particularly in the case of gene absence or erosion, these convergent patterns suggest relaxed selective pressure on gene function, rather than chance loss. These independent events may reflect the historical contingency of gene loss and erosion in the ancestral genome, which affects selective pressure on remaining genes.

We found frameshift errors in three genes involved in DNA replication or protein synthesis: $d n a G, d n a X$ and $p t h$. These genes are considered essential for fundamental cellular processes in E. coli. Although expression of full-length proteins may be restored by transcriptional slippage, previous work showed that full-length transcripts accounted for $₫ 30 \%$ of the transcripts produced from frameshifted genes [15]. Therefore, it is possible that frameshifts lower the effective expression of these genes in B. vafer. The impact on DNA replication fidelity and efficiency of protein synthesis is unclear.

The deletion hotspot detected around the putative origin of replication in both $B$. vafer and B. floridanus occurs in the $32 \mathrm{~kb}$ region between the $B$. vafer origin and the location of the origin in B. pennsylvanicus. Given that the putative origin of replication in B. pennsylvanicus occurs at the same genomic location as the origins in $E$. coli and in other insect endosymbionts of the gamma-proteobacteria, it seems likely that the origin of replication shifted in the ancestral lineage of $B$. vafer and $B$. floridanus. Although the mechanisms underlying this shift are unclear, it is interesting that relocation of the origin is correlated with a concentration of gene deletions in this region.

\section{Conclusions}

Comparisons of the $B$. vafer genome with the previously published B. floridanus and B. pennsylvanicus genomes clarified the core set of genes and functions shared by this mutualist group. More broadly, these data inform studies of processes of genome reduction in intracellular bacteria. (i) The surprising loss of glutamine synthetase $(g \ln A)$ from $B$. vafer, despite retention of urease genes, suggests a profound difference in nitrogen recycling within this species. In B. floridanus and B. pennsylvanicus, the ammonia generated by urease is assimilated during glutamine biosynthesis. In the C. vafer-Blochmannia association, ammonia assimilation may occur by an alternative pathway, such as glutamine biosynthesis in the host or the initial steps of arginine biosynthesis catalyzed by the endosymbiont. Database searches 
showed that lack of ammonia assimilation by glutamine synthetase or glutamate dehydrogenase in a ureaseencoding bacterium is extremely rare. (ii) Evaluating Blochmannia genome reduction in a phylogenetic context revealed instances of convergent gene loss or erosion at queA, visC, and $y g g S$, which may be associated with changes in selective pressure on these genes. (iii) A deletion hotspot in both B. vafer and B. floridanus occurs within a $32 \mathrm{~kb}$ region corresponding to the likely relocation of the replication origin. (iv) Eight B. vafer genes contain frameshifts in homopolymeric tracts, including two genes encoding DNA replication proteins and one gene important for efficient protein synthesis. Although such frameshifts may be corrected by transcriptional slippage, expression of full-length proteins is likely reduced compared to wild-type levels and has possible implications for efficiency and accuracy of information transfer.

\section{Methods}

\section{Blochmannia vafer genomic DNA preparation}

B. vafer genomic DNA (gDNA) was prepared from $C$. vafer workers and larvae collected from a single colony (Cvafe543) in the Coronado National Forest near Portal, Arizona. Vouchers from this colony will be deposited at the Bohart Museum of Entomology, University of California, Davis. We isolated Blochmannia cells from $7.8 \mathrm{~g}$ of ants using a Percoll density gradient centrifugation protocol described previously [37], with a $100 \mu \mathrm{m}$ filtration step instead of the $90 \mu \mathrm{m}$ filtration. Isolated Blochmannia cells were treated with DNase to remove extracellular host DNA, lysozyme and proteinase $\mathrm{K}$ to break down bacterial cell walls and membranes, and RNase to remove rRNA and other RNA species. After these treatments, we extracted gDNA with phenol chloroform followed by ethanol precipitation.

\section{Genome sequencing and assembly}

A paired-end sequencing library was constructed at Illumina (Hayward, CA) following standard procedures. Adaptors were used to multiplex this sample with two other samples on a single Illumina lane. Sequencing on an Illumina Genome Analyzer II (GAII) with Illumina pipeline 1.4 produced $14,907,136100$-bp paired end reads.

We performed all data analysis in house. Prior to de novo assembly, we filtered the read dataset to remove read pairs in which either read had an ambiguous base call (denoted by $\mathrm{N}$ ) or an average quality score «Q25. The filter retained $10,157,316$ reads (68\% of total read dataset). From these filtered reads, we randomly selected 3 million read pairs. We then assembled the reads with Velvet version 0.7.55 [38] using the following parameters: hash length 49 , expected coverage 200, coverage cutoff 25 and no scaffolding. This assembly strategy generated three contigs of Blochmannia genome sequence comprising 42,053 bp, 118,857 bp and 561,103 bp.

To close the three gaps, we first determined gap sizes by PCR amplification of the original gDNA using primers annealing to flanking regions in the assembled contigs. We closed the gap corresponding to the membrane-spanning repeat region of tolA with Sanger sequencing, using Phred/Phrap/Consed [39-41] to assemble and manually examine the sequence. Although the remaining two gaps were short, secondary structure arising from long palindromes impaired Sanger sequencing. Upon inspection, we found that palindromes had also impeded Velvet assembly, but the gaps could be manually closed with existing Illumina sequence data (Additional File 4).

To generate the finished genome, we used Mosaik [42] to align the full Illumina read dataset against the closed sequence. This step clarified the majority genotype (i.e., for each nucleotide position, the base called in the majority of reads), since de novo assembly used a subset of reads rather than the full read dataset. The Mosaik DupSnoop module identified $42 \%$ of the aligned read pairs as duplicates. Because most of these duplicates were likely generated during PCR amplification of the Illumina library, we removed duplicates from the final Mosaik alignment. We used Consed to view the alignment and recall the consensus sequence. Coverage of the finished genome (without duplicate read pairs) averaged $542 \times$, calculated using the MosaikCoverage module. The finished genome is deposited in GenBank with the accession number CP002189.

\section{Gene prediction and annotation}

The finished genome was submitted to the MANATEE annotation engine hosted by the Institute for Genome Sciences (IGS) at the University of Maryland School of Medicine for an initial automated annotation. MANATEE uses Glimmer for gene prediction, tRNAscan to identify tRNAs, and RNAmmer or Rfam to identify structural RNAs.

We manually curated the MANATEE annotation. An ORF was retained if it had at least one of the following pieces of evidence: a Blast-Extend-Repraze (BER) alignment scoring $<10^{-5}$ to a protein from another organism, a HMM hit or a Prosite hit. We used the gene name suggested by SwissProt for the homologous gene in $E$. coli to provide consistency with other proteobacterial genome annotations. For genes with no suggested name in SwissProt, such as hypothetical genes, the gene name given for the homologous gene in $B$. floridanus or $B$. pennsylvanicus was used.

We curated start sites on the basis of BER alignments to other, non-Blochmannia genomes. BER searches also 
identified genes that contain a frameshift within a polyA or polyT tract but otherwise resemble intact genes. Because these frameshifts may be corrected by transcriptional slippage, we consider these genes potentially functional and annotated them as intact ORFs. The GenBank annotation indicates the position of such frameshifts.

We used two approaches to identify uncalled genes and pseudogenes in B. vafer. First, we searched intergenic regions at least $30 \mathrm{bp}$ in length using BLASTX and the NCBI Protein Reference Sequences database with default parameters except that the low complexity sequence filter was turned off. We considered hits covering at least $60 \%$ of the subject protein sequence and yielding an e-score of $<10^{-5}$ as evidence of an uncalled gene or a pseudogene. For two intergenic regions, the top BLASTX hits were B. pennsylvanicus and/or B. floridanus, but the alignments had multiple nonsense mutations and gaps. Based on the alignments and their location within the conserved gene order of Blochman$n i a$, we identified these regions as pseudogenes of $b c p$ and queA.

We also took advantage of synteny in Blochmannia to screen for additional pseudogenes in B. vafer. For any genes present in B. floridanus and/or B. pennsylvanicus but missing in $B$. vafer, we compared the corresponding $B$. vafer intergenic region to the gene in the other Blochmannia genome(s) using TBLASTX. Apart from $b c p$ and queA as noted above, we found no sequence similarity pointing to other recognizable pseudogenes in B. vafer.

We used the tRNAscan-SE program to identify tRNAs in the genome [43]. This program flags a tRNA as a potential pseudogene if it has a primary structure (HMM) score $<10$ bits or a secondary structure score $<5$ bits. (The primary structure score reflects nucleotide sequence similarity to sequence profiles of tRNAs, whereas the secondary structure score reflects RNA folding into the characteristic cloverleaf structure.) Three tRNAs in B. vafer were flagged due to low primary structure scores. However, their secondary structure scores were 28, 33 and 43, well above the 5 bit cutoff, and the predicted secondary structures had the characteristic cloverleaf form. Because endosymbiont genomes have extremely low GC content, the primary structure score cutoff normally used for bacteria with more moderate GC content, such as E. coli, may not be appropriate for Blochmannia. Our examination of the three tRNAs flagged as possible pseudogenes suggest that these are functional tRNAs, and we have annotated them as such. Re-analysis of the B. pennsylvanicus genome, which has one reported pseudo-tRNA, revealed the same phenomenon of low primary structure score but high secondary structure score, therefore we consider this tRNA functional in this study.

\section{Screening for $g \ln A$ in additional $B$. vafer}

We confirmed the absence of glutamine synthetase $(g \ln A)$ in B. vafer from C. vafer workers collected from eight additional colonies in the Coronado National Forest. From a total DNA prep of an individual worker, we amplified a region of the $B$. vafer genome using primers anchored in $g m k$ and polA, which flank $r p o Z$ and $g \ln A$ in $B$. floridanus and $B$. pennsylvanicus. The resulting amplicons include the complete coding sequence of rpo $Z$ and the intergenic sequence corresponding to the former location of $g \ln A$. We sequenced the amplified region using Sanger sequencing and assembled the reads with Phred/Phrap/Consed [39-41]. We also sequenced trpE from all of these B. vafer except strain 21. Accession numbers are: HQ593629-HQ593636 (rpoZ region) and HQ603678-HQ603684 (trpE).

\section{Identification of $B$. vafer replication origin}

We used the Pattern Locator program [44] to search the $B$. vafer genome for $d n a A$ boxes using the consensus sequence TTWTNCACA [45]. In the absence of a characteristic cluster of $d n a A$ boxes, we used the CGview Server [46] to analyze the GC skew of the genome. The CGView Server calculates GC skew as $(\mathrm{G}-\mathrm{C}) /(\mathrm{G}+\mathrm{C})$ using sliding windows of $1000 \mathrm{bp}$ and a step size of 10 bp. The CGView Server also calculated GC content using the same sliding window parameters.

\section{Palindrome analysis}

We used the EMBOSS palindrome script to search the genomes of B. vafer, B. floridanus, and B. pennsylvanicus for palindromes between $30-1000 \mathrm{bp}$, with a maximum gap of $20 \mathrm{bp}$ between inverted repeats and a maximum of 3 mismatches.

\section{Comparisons among Blochmannia genomes (i) Gene content comparisons}

We compared the gene content of the three Blochmannia genomes using a bidirectional best hit BLASTP analysis available through RAST (Rapid Annotation using Subsystem Technology) [47]. Pairwise comparisons of the genomes identified shared genes as bidirectional best hits. For genes yielding unidirectional best hits or fragmented hits, such as genes with frameshifts in homopolymeric tracts, we examined the annotations and performed additional BLASTP analyses to determine the presence or absence of the gene.

\section{(ii) Detection of additional B. floridanus and B. \\ pennsylvanicus genes}

Based on comparisons of all three sequenced Blochmannia, we identified four protein-coding genes in B. floridanus and a RNA-coding gene in both B. floridanus and $B$. pennsylvanicus that were not previously annotated. We included these genes in our analyses here. To 
determine the start and stop coordinates of the proteincoding genes, we used TBLASTN to align the B. vafer or $B$. pennsylvanicus amino acid sequence to the $B$. floridanus nucleotide sequence. If the alignment did not include start and stop codons, we extended the coordinates to include the closest upstream ATG start codon and the downstream stop codon. To determine the coordinates of the RNA-coding gene, we used the Rfam sequence search.

\section{(iii) Analysis of frameshifted genes}

Genes containing single frameshifts within polyA or polyT tracts were compared to orthologs in the other Blochmannia genomes. Nucleotide sequences were translated, aligned with ClustalX and back-translated to obtain nucleotide sequence alignments. For the purposes of comparing evolutionary rates at these loci, the frameshifts within polyA tracts were corrected, and the genes were treated as intact.

\section{(iv) Evolutionary rate comparison}

We compared rates of protein evolution in the lineages leading to B. vafer and B. floridanus, using B. pennsylvanicus as an outgroup. First, we used the Reciprocal Smallest Distance (RSD) algorithm [48] to identify orthologs shared among the three genomes. By using stringent detection parameters in RSD, we included only those orthologs with robust alignments for $\mathrm{dN}$ calculations. (Reflecting the stringent criteria, RSD detected slightly fewer shared orthologs (570) than the 575 protein-coding genes detected in our direct comparisons of gene contents.) For each shared ortholog, we estimated the three pairwise $\mathrm{dN}$ values using a likelihood-based approach in PAML [49]. We then calculated the fold-increase in evolutionary rate for each gene along the lineage leading to $B$. vafer as $\mathrm{K}_{1} / \mathrm{K}_{2}$, in which $\mathrm{K}_{1}$ is the amount of nonsynonymous change along the branch leading to $B$. vafer from the most recent common ancestor with $B$. floridanus, and $\mathrm{K}_{2}$ is the amount of nonsynonymous change along the branch leading to $B$. floridanus.

\section{(v) IGS analysis}

We defined intergenic spacer sequences (IGS) as the sequences between protein-coding genes, RNA-coding genes or pseudogenes. Our calculation of IGS lengths does not include instances of overlapping gene coordinates that result in no intergenic sequence between the two genes. We used a perl script written by Sheri Simmons to extract intergenic sequences from the $B$. vafer genome.

Comparison of gene content across insect endosymbionts Among select other bacterial endosymbionts, we checked for the presence of protein-coding genes that were missing or eroded in the Blochmannia genomes. For each gene, the protein sequence from E. coli K12 MG1655 (NC_000913) was used as the query in a TBLASTN search against the endosymbiont genomes. For a gene to qualify as present, the alignment must cover at least $60 \%$ of the $E$. coli protein sequence and have an e-score $<10^{-5}$ (with the exception of fts $Y$, which required at least $50 \%$ coverage and an e-score $\left\langle 10^{-5}\right.$ ). We examined the annotations of the endosymbiont genomes to ensure that the hits corresponded to homologous genes, rather than a similar gene from the same family (such as isp $A$ and ispB). If the region corresponding to the alignment was not annotated in the endosymbiont genome, we did a BLASTP analysis of the encoded amino acid sequence against the SwissProt database and used the top hit to identify the gene.

\section{Analysis of urease and ammonia assimilation enzyme distribution in bacterial genomes}

We used the $B$. pennsylvanicus ure $C$ amino acid sequence in a TBLASTN search of the NCBI Genomes database with TaxID 2 to limit the search to bacterial genomes. This search returned 371 hits with at least $50 \%$ coverage and an e-score $<10^{-30}$. We then did a TBLASTN search of these 371 bacterial genomes using the B. pennsylvanicus $g \ln A$ amino acid sequence. The gene was considered present if the alignment had at least 50\% coverage and an e-score $<10^{-30}$. For three genomes, we discovered that $u r e C$ and $g \ln A$ were encoded on different chromosomes, and for one genome, ure $C$ was encoded on a plasmid whereas $g \ln A$ was encoded on the chromosome. Genomes that did not encode an ortholog of $B$. pennsylvanicus glnA were searched for Class III glutamine synthetase from Bacteroides fragilis $\mathrm{YCH} 46$ (NC_006347) and glutamate dehydrogenase from E. coli K12 MG1655 (NC_000913) using TBLASTN.

\section{Statistical analysis}

All statistical analyses used JMP version 8.

\section{Additional material}

Additional File 1: Ortholog table of the three Blochmannia

genomes. Table listing the annotated gene sets for all three available Blochmannia genomes.

Additional File 2: Evolutionary rates analysis. Table listing the complete results of the evolutionary rates comparison.

Additional File 3: Phylogenetic analysis of Blochmannia vafer Description of phylogenetic analyses of the three sequenced Blochmannia.

Additional File 4: Gap closure. Detailed methods used to close two gaps in the B. vafer genome assembly.

\footnotetext{
Acknowledgements

The authors gratefully acknowledge the assistance of Michelle Giglio, Todd Creasy and Sean Daughtery at the Institute for Genome Sciences at the University of Maryland School of Medicine for MANATEE implementation. We thank several colleagues at the Marine Biological Lab: Adam Lazarus collected ant specimens and obtained Blochmannia trpE sequences, James Style prepared genomic DNA, Rich Fox installed and compiled many of the bioinformatics tools used here, Sheri Simmons provided the script for
} 
extracting intergenic sequences, and Gretta Serres offered helpful discussions of functional annotations. Nan Leng at Illumina, Inc. provided the average quality score filtering script. We also thank four anonymous reviewers for their excellent comments and suggestions. This work was supported by grants to JJW from the NSF (MCB-0604177) and NIH (R01GM062626).

\section{Author details}

The Institute for Genome Sciences and Policy, Duke University, Durham, NC, USA. ${ }^{2}$ Nicholas School of the Environment, Duke University, Durham, NC, USA.

\section{Authors' contributions}

LEW assembled and annotated the genome and performed comparative analyses. JJW conceived the study and performed the evolutionary rate comparison. LEW and JJW designed the comparative analyses and wrote the manuscript. Both authors read and approved the final manuscript.

Received: 16 September 2010 Accepted: 2 December 2010 Published: 2 December 2010

\section{References}

1. Sauer C, Stackebrandt E, Gadau J, Holldobler B, Gross R: Systematic relationships and cospeciation of bacterial endosymbionts and their carpenter ant host species: proposal of the new taxon Candidatus Blochmannia gen. nov. Int J Syst Evol Microbiol 2000, 50(Pt 5):1877-1886.

2. Blochman F: Über das Vorkommen von bakterienähnlichen Gebilden in den Geweben und Eiern verschiedener Insekten. Zentbl Bakteriol 1882, 11:234-240.

3. Wernegreen JJ, Kauppinen SN, Brady SG, Ward PS: One nutritional symbiosis begat another: phylogenetic evidence that the ant tribe Camponotini acquired Blochmannia by tending sap-feeding insects. BMC Evol Biol 2009, 9:292

4. Schroder D, Deppisch H, Obermayer M, Krohne G, Stackebrandt E, Holldobler B, Goebel W, Gross R: Intracellular endosymbiotic bacteria of Camponotus species (carpenter ants): Systematics, evolution and ultrastructural characterization. Mol Microbiol 1996, 21(3):479-489.

5. Buchner P: Endosymbiosis of Animals with Plant Microorganisms New York: Interscience Publishers, Inc; 1965.

6. Gil R, Silva FJ, Zientz E, Delmotte F, Gonzalez-Candelas F, Latorre A, Rausell C, Kamerbeek J, Gadau J, Holldobler B, et al: The genome sequence of Blochmannia floridanus: comparative analysis of reduced genomes. Proc Natl Acad Sci USA 2003, 100(16):9388-9393.

7. Degnan PH, Lazarus AB, Wernegreen JJ: Genome sequence of Blochmannia pennsylvanicus indicates parallel evolutionary trends among bacterial mutualists of insects. Genome Res 2005, 15(8):1023-1033.

8. Feldhaar H, Straka J, Krischke M, Berthold K, Stoll S, Mueller MJ, Gross R: Nutritional upgrading for omnivorous carpenter ants by the endosymbiont Blochmannia. BMC Biol 2007, 5:48.

9. Zientz E, Dandekar T, Gross R: Metabolic interdependence of obligate intracellular bacteria and their insect hosts. Microbiol Mol Biol Rev 2004, 68(4):745-770.

10. Moran NA, McCutcheon JP, Nakabachi A: Genomics and evolution of heritable bacterial symbionts. Annu Rev Genet 2008, 42:165-190.

11. van Ham RC, Kamerbeek J, Palacios C, Rausell C, Abascal F, Bastolla U, Fernandez JM, Jimenez L, Postigo M, Silva FJ, et al: Reductive genome evolution in Buchnera aphidicola. Proc Natl Acad Sci USA 2003, 100(2):581-586.

12. Mira A, Ochman $\mathrm{H}$, Moran NA: Deletional bias and the evolution of bacterial genomes. Trends Genet 2001, 17(10):589-596.

13. Pal C, Papp B, Lercher MJ, Csermely P, Oliver SG, Hurst LD: Chance and necessity in the evolution of minimal metabolic networks. Nature 2006, 440(7084):667-670.

14. Moran NA, McLaughlin HJ, Sorek R: The dynamics and time scale of ongoing genomic erosion in symbiotic bacteria. Science 2009, 323(5912):379-382

15. Tamas I, Wernegreen JJ, Nystedt B, Kauppinen SN, Darby AC, GomezValero L, Lundin D, Poole AM, Andersson SG: Endosymbiont gene functions impaired and rescued by polymerase infidelity at poly(A) tracts. Proc Natl Acad Sci USA 2008, 105(39):14934-14939.
16. Wernegreen JJ, Kauppinen SN, Degnan PH: Slip into something more functional: selection maintains ancient frameshifts in homopolymeric sequences. Mol Biol Evol 2010, 27(4):833-839.

17. Bolton B, Alpert G, Ward PS, Naskrecki P: Bolton's Catalogue of Ants of the World: 1758-2005. Cambridge, MA: Harvard University Press; 2006.

18. Snelling RR: Taxonomy of the Camponotus festinatus complex in the United States of America (Hymenoptera: Formicidae). Myrmecologische Nachrichten 2006, 8:83-97.

19. Lee MH, Mulrooney SB, Renner MJ, Markowicz Y, Hausinger RP: Klebsiella aerogenes urease gene cluster: sequence of $u r e D$ and demonstration that four accessory genes (ureD, ureE, ureF, and ureG) are involved in nickel metallocenter biosynthesis. J Bacteriol 1992, 174(13):4324-4330.

20. Reitzer L: Nitrogen assimilation and global regulation in Escherichia coli. Annu Rev Microbiol 2003, 57:155-176.

21. Smith DG, Russell WC, Ingledew WJ, Thirkell D: Hydrolysis of urea by Ureaplasma urealyticum generates a transmembrane potential with resultant ATP synthesis. J Bacteriol 1993, 175(11):3253-3258.

22. Blinkowa $A L$, Walker JR: Programmed ribosomal frameshifting generates the Escherichia coli DNA polymerase-III gamma subunit from within the gamma-subunit reading frame. Nucleic Acids Res 1990, 18(7):1725-1729.

23. Flower AM, Mchenry CS: The gamma-subunit of DNA polymerase-III holoenzyme of Escherichia coli is produced by ribosomal frameshifting. Proc Natl Acad Sci USA 1990, 87(10):3713-3717.

24. Tsuchihashi Z, Kornberg A: Translational frameshifting generates the gamma-subunit of DNA Polymerase-III holoenzyme. Proc Natl Acad Sci USA 1990, 87(7):2516-2520

25. McHenry CS: Chromosomal replicases as asymmetric dimers: studies of subunit arrangement and functional consequences. Mol Microbiol 2003, 49(5):1157-1165

26. Das G, Varshney U: Peptidyl-tRNA hydrolase and its critical role in protein biosynthesis. Microbiology 2006, 152(Pt 8):2191-2195.

27. Williams KP, Gillespie JJ, Sobral BWS, Nordberg EK, Snyder EE, Shallom JM Dickerman AW: Phylogeny of Gammaproteobacteria. J Bacteriol 2010, 192(9):2305-2314

28. Nakamura T, Katoh Y, Shimizu Y, Matsuba Y, Unemoto T: Cloning and sequencing of novel genes from Vibrio alginolyticus that support the growth of $\mathrm{K}+$ uptake-deficient mutant of Escherichia coli. Biochim Biophys Acta 1996, 1277(3):201-208

29. Ito T, Uozumi N, Nakamura T, Takayama S, Matsuda N, Aiba H, Hemmi H, Yoshimura T: The implication of YggT of Escherichia coli in osmotic regulation. Biosci, Biotechnol, Biochem 2009, 73(12):2698-2704.

30. Treangen TJ, Abraham AL, Touchon M, Rocha EP: Genesis, effects and fates of repeats in prokaryotic genomes. FEMS Microbiol Rev 2009, 33(3):539-571

31. Gomez-Valero L, Latorre A, Gil R, Gadau J, Feldhaar H, Silva FJ: Patterns and rates of nucleotide substitution, insertion and deletion in the endosymbiont of ants Blochmannia floridanus. Mol Ecol 2008.

32. Bonasio R, Zhang G, Ye C, Mutti NS, Fang X, Qin N, Donahue G, Yang P, Li Q, Li C, et al: Genomic comparison of the ants Camponotus floridanus and Harpegnathos saltator. Science 2010, 329(5995):1068-1071.

33. Rubino SD, Nyunoya H, Lusty CJ: In vivo synthesis of carbamyl phosphate from NH3 by the large subunit of Escherichia coli carbamyl phosphate synthetase. J Biol Chem 1987, 262(9):4382-4386.

34. Lopez-Sanchez MJ, Neef A, Pereto J, Patino-Navarrete R, Pignatelli M, Latorre A, Moya A: Evolutionary convergence and nitrogen metabolism in Blattabacterium strain Bge, primary endosymbiont of the cockroach Blattella germanica. PLoS Genet 2009, 5(11):e1000721.

35. Sabree ZL, Kambhampati S, Moran NA: Nitrogen recycling and nutritional provisioning by Blattabacterium, the cockroach endosymbiont. Proc Nat Acad Sci USA 2009, 106(46):19521-19526.

36. Glass JI, Lefkowitz EJ, Glass JS, Heiner CR, Chen EY, Cassell GH: The complete sequence of the mucosal pathogen Ureaplasma urealyticum. Nature 2000, 407(6805):757-762.

37. Wernegreen JJ, Lazarus AB, Degnan PH: Small genome of Candidatus Blochmannia, the bacterial endosymbiont of Camponotus, implies irreversible specialization to an intracellular lifestyle. Microbiology 2002, 148(Pt 8):2551-2556.

38. Zerbino DR, Birney E: Velvet: Algorithms for de novo short read assembly using de Bruijn graphs. Genome Res 2008, 18(5):821-829.

39. Gordon D, Abajian C, Green P: Consed: a graphical tool for sequence finishing. Genome Res 1998, 8(3):195-202. 
40. Ewing B, Green P: Base-calling of automated sequencer traces using phred. II. Error probabilities. Genome Res 1998, 8(3):186-194.

41. Ewing B, Hillier L, Wendl MC, Green P: Base-calling of automated sequencer traces using phred. I. Accuracy assessment. Genome Res 1998, 8(3): 175-185.

42. Mosaik. [http://bioinformatics.bc.edu/marthlab/Mosaik].

43. Lowe TM, Eddy SR: tRNAscan-SE: a program for improved detection of transfer RNA genes in genomic sequence. Nucleic Acids Res 1997, 25(5):955-964.

44. Mrazek J, Xie SH: Pattern locator: a new tool for finding local sequence patterns in genomic DNA sequences. Bioinformatics 2006, 22(24):3099-3100.

45. Schaper S, Messer W: Interaction of the initiator protein DnaA of Escherichia coli with Its DNA target. J Biol Chem 1995, 270(29):17622-17626

46. Grant JR, Stothard P: The CGView Server: a comparative genomics tool for circular genomes. Nucleic Acids Res 2008, 36:W181-W184.

47. Aziz RK, Bartels D, Best AA, DeJongh M, Disz T, Edwards RA, Formsma K, Gerdes S, Glass EM, Kubal M, et al: The RAST server: rapid annotations using subsystems technology. BMC Genomics 2008, 9:75.

48. Wall DP, Fraser HB, Hirsh AE: Detecting putative orthologs. Bioinformatics 2003, 19(13):1710-1711.

49. Yang Z: PAML: a program package for phylogenetic analysis by maximum likelihood. Comput Appl Biosci 1997, 13(5):555-556.

\section{doi:10.1186/1471-2164-11-687}

Cite this article as: Williams and Wernegreen: Unprecedented loss of ammonia assimilation capability in a urease-encoding bacterial mutualist. BMC Genomics 2010 11:687.

\section{Submit your next manuscript to BioMed Central and take full advantage of:}

- Convenient online submission

- Thorough peer review

- No space constraints or color figure charges

- Immediate publication on acceptance

- Inclusion in PubMed, CAS, Scopus and Google Scholar

- Research which is freely available for redistribution

Submit your manuscript at www.biomedcentral.com/submit 